\title{
Characteristics, Risk Factors, and Prevalence of Clostridioides difficile Among Hospitalized Patients in a Tertiary Care Hospital in Palestine
}

\author{
Abdallah Damin Abukhalil' \\ Liana AbuKhdeir' \\ Malak Hamed' \\ Ni'meh Al Shami (D)' \\ Hani A Naseef' \\ Banan M Aiesh ${ }^{2}$ \\ Ali Sabateen ${ }^{2}$ \\ 'Pharmacy Department, Faculty of \\ Pharmacy, Nursing and Health \\ Professions, Birzeit University, Birzeit, \\ West Bank, Palestine; ${ }^{2}$ An-Najah National \\ University Hospital, Nablus, Palestine
}

\begin{abstract}
Purpose: Clostridioides difficile is one of the most important nosocomial infection pathogens. It is linked with many risk factors. Unfortunately, many studies have been conducted in different countries to address the Clostridioides difficile infections (CDI), and no studies have been conducted in Palestine. This study aims to identify the prevalence and possible risk factors associated with CDI.

Patients and Methods: This was a retrospective descriptive study conducted at the AnNajah National University Hospital (NNUH) in Palestine. Data were collected for patients diagnosed with CDI who tested positive for GDH, toxins A and B between January 2018 and April 30, 2021. In addition, patient characteristics and risk factors associated with CDI were analyzed.

Results: A total of 593 participants were included in the study; 53\% had hospital-acquired CDI. There was an insignificant association between participant age and CDI risk. Most patients had mild to moderate infections. Sixty-three percent of the participants were immunocompromised. About 58.5\% used an antibiotic agent two weeks before CDI, and $67 \%$ were on a proton pump inhibitor (PPI). About $61.3 \%$ of patients were treated according to IDSA 2017 guidelines, and 94\% responded adequately to the treatment provided.
\end{abstract}

Conclusion: There was an increased prevalence of community-acquired CDI, with a prevalence almost equal to that of hospital-acquired. In addition, most of the participants were immunocompromised. The risk factors for CDI, such as antibiotics and PPI use, were also observed with high prevalence among positive patients. Antimicrobial stewardship and the appropriate use of acid suppressors are warranted.

Keywords: Clostridium difficile infection, Clostridium difficile risk factors, communityacquired CDI, hospital-acquired CDI, PPI, antibiotics

\section{Introduction}

Clostridioides difficile is a gram-positive spore-forming anaerobic bacterium that causes gastrointestinal tract infection due to disruption of the normal microbiota of the colon caused by antimicrobial agents. CDI has been associated with the most nosocomial diarrheal infections among hospitalized patients, ${ }^{1}$ causing substantial public health threats associated with higher morbidity and mortality worldwide. ${ }^{2}$

Symptoms related to CDI are associated with the production and release of different toxins. ${ }^{3}$ Symptoms of CDI include watery diarrhea (at least three watery stools within 24 hours), abdominal pain, fever and nausea. C. difficile is transmitted from person to person via the fecal-oral route. Healthcare facilities are at the highest
Correspondence: Abdallah Damin Abukhalil

Pharmacy Department, Faculty of Pharmacy, Nursing and Health Professions, Birzeit University, P.O. Box 14, Birzeit, West Bank, Palestine Tel $+970-598204036$

Fax +970-2-2982017

Email Adkhalil@birzeit.edu 
risk of infection transmission due to environment, surfaces, and health worker contamination. Therefore, good hygiene among healthcare workers and surface disinfection are essential to minimize CDI. In addition, CDI patients must be isolated from other patients to prevent transmission.

$\mathrm{CDI}$ is classified as community-acquired (CA) or hospital-acquired (HA). According to the Infectious Disease Society of America (IDSA) guidelines, CDI is considered community-acquired if the diagnosis was without exposure to the healthcare facility after 12 weeks of discharge. On the other hand, it is regarded as hospital-acquired if the diagnosis was after 3 days of admission or within four weeks of release. ${ }^{4}$

Several factors increase the likelihood of CDI, including antibiotic exposure, older age, and hospitalization. ${ }^{1}$ Antibiotic use and previous exposure to $C$. difficile are the two main risk factors for CDI. Fluoroquinolones, cephalosporins, carbapenems, and clindamycin have the highest risk of CDI. ${ }^{5,6}$ Other potential risk factors include; stomach acid suppression medications, immunocompromised patients, irritable bowel syndrome and chronic kidney disease, prolonged exposure to healthcare facilities, and enteral feeding (tube feeding) have the potential to cause CDI. $^{7,8}$

Treatment for CDI includes vancomycin, fidaxomicin, and metronidazole. The most recent guidelines update in 2017 by the IDSA and the society for healthcare epidemiology (SHEA) recommend vancomycin or fidaxomicin as first-line agents for initial treatment of CDI while metronidazole is preserved as an alternative therapy. ${ }^{4}$

During the last few years, there has been an increase in CDI rates due to the emergence of new strains. ${ }^{9}$ According to the Centers for Disease Control and Prevention (CDC), approximately half a million infections are estimated to occur in the United States each year. In addition, according to the National Institutes of Health (NIH), CDI increases morbidity, mortality, and healthcare costs. Hence, the total annual $C$. difficile attributable cost in the United States is approximately $\$ 6.3$ billion.

Minimal data are available on the prevalence and characteristics of CDI infections in Palestine. Therefore, this retrospective descriptive study aimed to determine CDI prevalence among hospitalized patients in a Palestinian teaching hospital by examining infection characteristics, risk factors, comorbidities, and treatment.

\section{Methods}

A retrospective descriptive study at the An-Najah National University Hospital (NNUH) in Palestine was conducted for all patients diagnosed with CDI from January 2018 to April 30, 2021. A laboratory-confirmed CDI was a patient with positive ELISA test detection of A or B CDI toxins. The CoproStrep C. difficle GDH + Toxin A + Toxin $B$ rapid test was used to aid in CDI diagnosis. Patients with positive antigen results and negative toxin $\mathrm{A}$ or $B$ results were excluded from the study due to the lack of other tests such as PCR for test result confirmation. In addition, patients who had laboratory test results with incomplete patient documentation or records were also excluded from the study.

Data on patient information, diagnosis, treatment, comorbidities, risk factors, and symptoms associated with CDI were collected and analyzed using the Statistical Package for Social Sciences (SPSS) version 22. Data collected included patient demographic information, hospitalization length, CDI symptoms such as diarrhea and abdominal pain, and the type of CDI community or hospital acquired. Prior antibiotics, proton pump inhibitors (PPIs), H2 receptor antagonists, and laxatives use were also collected. In addition, patients' comorbidities were considered and if the patient was immunocompromised. Data were recorded and categorized as required. Frequencies and percentages were used to represent the data. Pearson's Chi-square test was performed to assess the association between the possibility of being a CDinfected patient and patients' age and sex as potential risk factors.

\section{Ethical Committee}

This was a retrospective observational study conducted at the An-Najah National University Hospital (NNUH) in Palestine. The IRB committee approved the study design at Birzeit University and An-Najah Medical Hospital reference number BZUPNH2102. The requirement to obtain written informed consent from each patient was waived because this was an observational retrospective study. The patients were anonymized. Their information was nonidentifiable. This study complied with the ethical guidelines of the Declaration of Helsinki and patient data.

\section{Results}

Data from 593 patients were extracted from the files for the current study. Laboratory test results revealed that 105 $(17.7 \%)$ had a positive $C$. difficile antigen, 79 (13.3\%) had 
Table I Patient Characteristics of Clostridium difficile Toxins Test Result

\begin{tabular}{|c|c|c|c|c|c|}
\hline \multicolumn{2}{|c|}{ Characteristic } & \multirow{3}{*}{$\begin{array}{l}\text { Participants n(\%) } \\
281(47.4) \\
312(52.6)\end{array}$} & \multicolumn{2}{|c|}{ Toxin A/Toxin B Test Result } & \multirow{3}{*}{$\begin{array}{l}\text { P- value } \\
0.195\end{array}$} \\
\hline & & & Positive n(\%) & Negative $n(\%)$ & \\
\hline Sex & $\begin{array}{l}\text { Male } \\
\text { Female }\end{array}$ & & $\begin{array}{l}53(17) \\
37(13.2)\end{array}$ & $\begin{array}{l}259(83) \\
244(86.8)\end{array}$ & \\
\hline Age & $\begin{array}{l}2-18 \\
19-44 \\
45-64 \\
65 \text { or more }\end{array}$ & $\begin{array}{l}54(9.1) \\
195(32.9) \\
202(34.1) \\
142(23.9)\end{array}$ & $\begin{array}{l}13(24.1) \\
29(14.9) \\
30(14.9) \\
18(12.7)\end{array}$ & $\begin{array}{l}41 \text { (75.9) } \\
166(85.1) \\
172(85.1) \\
124(87.3)\end{array}$ & 0.257 \\
\hline
\end{tabular}

a positive toxin A test, and $77(13 \%)$ had a positive result for toxin B. A total of 90 patients had a positive result for toxin A or B; 8 patients were excluded due to incomplete records. In addition, 38 (46.9\%) were community-acquired infections, while $43(53.1 \%)$ were acquired due to hospital admission.

Half of the patients, 312 (52.6\%), were females. 53 (17\%) male and $37(13.2 \%)$ female patients had a positive result for the $C$. difficile toxin test, as shown in Table 1, with no significant difference between the two groups $(\mathrm{P}=$ 0.195) (Table 1).

Regarding age groups, findings demonstrated that most patients aged between 19 and 64 years with the highest percentage with positive CDI toxin test were among patients aged $2-18$ years $13(24.1 \%)$. No significant differences were found between the four age groups and their possibility of being infected $(\mathrm{P}=0.275)$ (Table 1$)$.

The characteristics, risk factors, and symptoms with a confirmed diagnosis are presented in Table 2. Among 82 patients, $58.5 \%, 65.8 \%, 4.9 \%, 7.3 \%$ had used antibiotics, PPIs, H2 antagonists, and laxatives during the six weeks before CDI, respectively. The percentages of patients who suffered from abdominal pain and diarrhea

Table 2 Participant's Characteristics and Symptoms $(\mathrm{N}=82)$

\begin{tabular}{|l|l|c|}
\hline Characteristics & & n (\%) \\
\hline Prior antibiotic use & Yes & $48(58.5 \%)$ \\
\hline PPI use & No & $28(34.2 \%)$ \\
& 4 days or less & $12(14.6 \%)$ \\
& $>4$ day & $42(52.4 \%)$ \\
\hline Anti H2 use & Yes & $4(4.9 \%)$ \\
Laxative & Yes & $6(7.3 \%)$ \\
Abdominal pain & Yes & $43(46.9 \%)$ \\
Diarrhea & Yes & $73(90.1 \%)$ \\
Treatment & Inpatient & $69(84.1 \%)$ \\
Immunocompromised & Yes & $52(63.4 \%)$ \\
\hline
\end{tabular}

were $46.9 \%$ and $90.1 \%$. In addition, $84.1 \%$ were treated as inpatients and $63.4 \%$ were immunocompromised.

Table 3 shows antibiotics administered within six weeks prior to the confirmation of CDI diagnosis. The antibiotics used most were Piperacillin-tazobactam 20 (24.1\%), aminoglycosides 19 (23.2\%), and cephalosporins 18 (22\%).

Of the 82 patients with a confirmed diagnosis of CDI, 80 had a complete record of the treatment regimen, 49 $(61.3 \%)$ had a treatment based on 2017 treatment guidelines (the latest guideline), while in 31 (38.7\%) of the cases, the treatment guidelines were not followed. In evaluating patients' outcomes, in one case $(1.5 \%)$, there was no indication for treatment, $75(93.8 \%)$ responded to therapy while $4(5 \%)$ were still untreated.

Among the total 82 patients with positive CDI results, $43 \%$ were found to have moderate CDI. $31.6 \%$ had mild, $16.5 \%$ had severe, and only $8.9 \%$ had Fulminant CDI (Figure 1).

The usage of antibiotics and PPIs among participants with $C$. difficile toxin tests was compared based on CDI severity. The results show that $84.6 \%$ of severe cases, more than $60 \%$ of mild and $50 \%$ of fulminant cases have used both antibiotics and a PPI before developing CDI (Figure 2).

Table 3 The Frequency of Antibiotics Used Prior Diagnosis $(\mathrm{N}=82)$

\begin{tabular}{|l|l|}
\hline Prior antibiotic use & n (\%) \\
\hline Piperacillin-tazobactam & $20(24.4 \%)$ \\
Aminoglycosides & $19(23.2 \%)$ \\
Cephalosporins & $18(22 \%)$ \\
Vancomycin & $18(22 \%)$ \\
Fluoroquinolones & $16(19.5 \%)$ \\
Carbapenems & $1 \mathrm{I}(13.4 \%)$ \\
Colistin & $5(6.1 \%)$ \\
Metronidazole & $3(3.7 \%)$ \\
Penicillin & $2(2.4 \%)$ \\
Clindamycin & $2(2.4 \%)$ \\
\hline
\end{tabular}




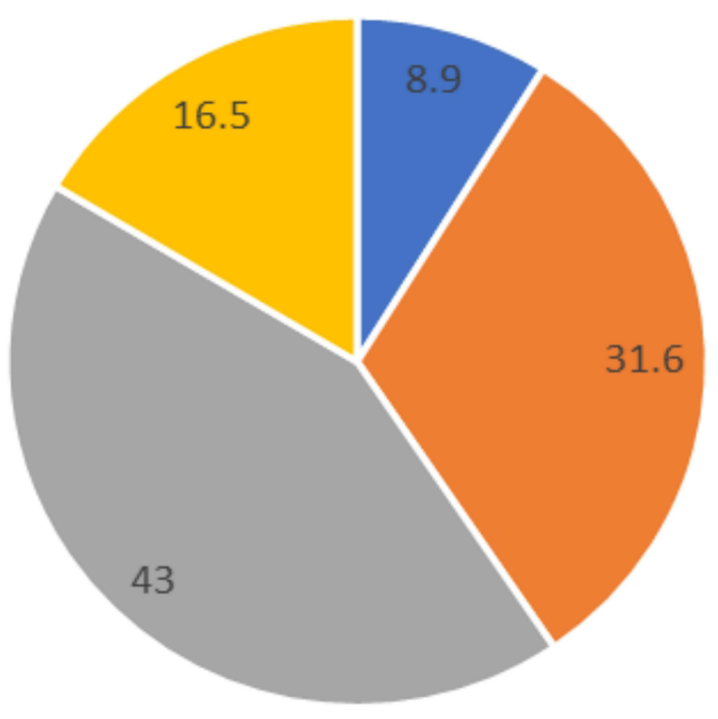

- Fulminant | Mild | Moderate - Severe

Figure I CDI classifications as percentages $(\mathrm{N}=82)$.

In this study, $93 \%$ of hospital-acquired CDI (HACDI) cases were treated with PPI, and $88.4 \%$ were also on antimicrobial agents. On the other hand, 36.8\% of the community-acquired CDI (CA-CDI) cases used PPI, and $23.7 \%$ had taken antibiotics prior to CDI (Figure 3).

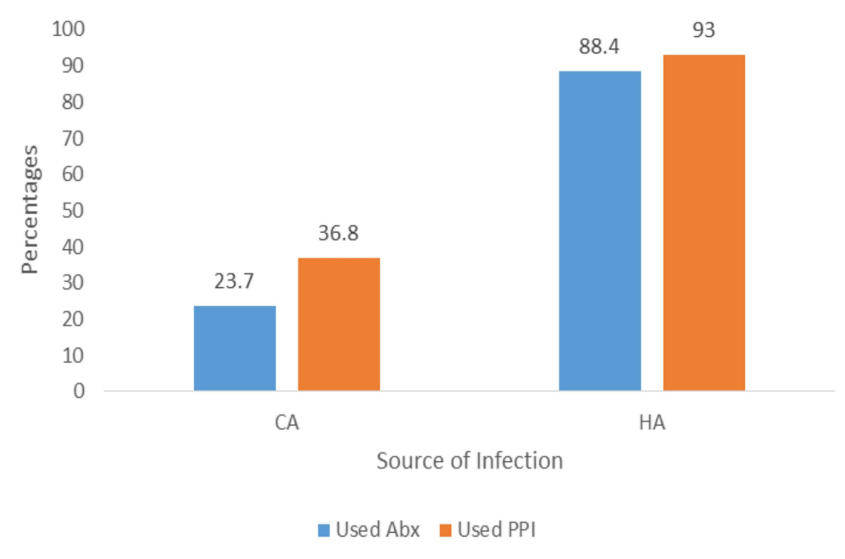

Figure $3 \mathrm{CA}-\mathrm{CDI} / \mathrm{HA}-\mathrm{CDI}$ and medication use $(\mathrm{N}=82)$.

\section{Discussion}

This study was the first to address CDI's prevalence and risk factors among hospitalized and admitted patients in a teaching hospital in Palestine. According to many studies conducted in different countries, CDI has always been associated with many risk factors. The main risk factors for $\mathrm{CDI}$ are antibiotic exposure, stomach acid suppression medications, increased age, type of feeding, and extended hospitalization. These factors were also very evident in the study population.

Three-year hospital admission data revealed a total of 593 patients suspected of having a CDI infection, with only 90 patients tested positive for CDI toxin, which represents a very low prevalence of CDI infection. In addition, the

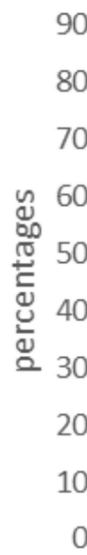

90

80

70

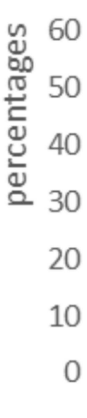

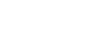

57.157 .1

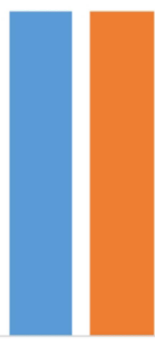

Fulminant

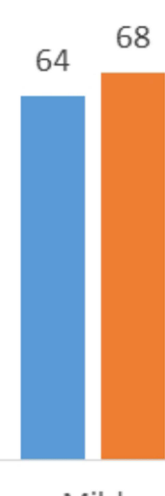

Mild
84.684 .6

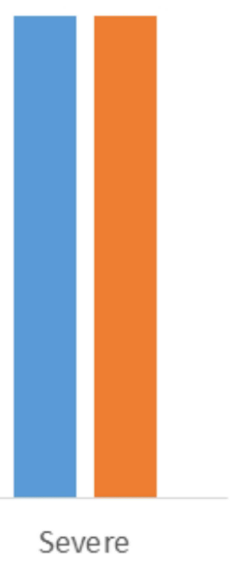

Moderate

Severe

\section{Classification}

Figure $2 \mathrm{CDI}$ classification and medication use $(\mathrm{N}=82)$. 
results of the CDI toxin test revealed no significant gender difference between male and female patients $p$-value 0.195 . The result is similar to the Michigan university study finding, showing no gender difference in prevalence among patients infected with CDI. ${ }^{9}$

A recent review in 2017 at the Center for AntiInfective Research and Development, Hartford Hospital, Hartford, USA, reported increased age as a risk factor for CDI. ${ }^{10}$ In 2015 the CDC reported that one out of every three CDIs occurred in patients aged 65 years or older. In addition, two out of every three healthcare-associated CDIs occurred in patients aged 65 years or older. ${ }^{8}$ Older ages are at increased risk for CDI due to the comorbidities usually seen in the elderly and physiological changes in the body, such as changes in immune system reactions and normal gastrointestinal flora. In contrast to the above, although the results indicated that the highest percentage with positive CDI toxin test $(24 \%)$ was among patients aged 2-18 years, there was no significant difference among different patients based on age. This finding disagrees with other studies or CDC reporting due to the social characteristics of the elderly in Palestine and the lack of nursing home utilization increasing the risk among the elderly. ${ }^{11}$ In addition, studies have shown that children are at an increased risk of developing CDI, especially community-acquired CDI. ${ }^{12,13}$

A Washington University School of Medicine study for risk factors within the elderly group showed that elderly individuals with other risk factors such as overall health status affect the patient's risk of CDI. ${ }^{14}$ Another study by the Memorial Sloan Kettering Cancer Center in New York revealed that patients with comorbidities, such as hematologic malignancies are at a higher risk of developing CDI. ${ }^{15}$ This finding is evident in our study, in which more than $63 \%$ of the diagnosed patients were immunocompromised. An Oxford University study suggests that immunocompromised patients experience a high incidence of CDI, ranging from $6 \%$ to $33 \%$ distributed over cancers, HIV patients and solid organ transplant recipients. ${ }^{16}$ Immunocompromised patients with suppressed immune systems cannot prevent or delay the binding of either the bacterium or the secreted toxins to the bowel tract epithelial cells where they can cause damage. ${ }^{17}$

The CDC reported that antibiotics are a well-known cause of CDI and increase the likelihood of a patient catching C. difficile. ${ }^{18}$ An analysis of the FDA Adverse Event Reporting System showed that patients who took penicillin combinations, carbapenems, cephalosporins, tetracyclines, macrolides, fluoroquinolones and trimethoprim-sulfamethoxazole were at higher risk of developing CDI. ${ }^{19}$ More than half of the participants in this study used antibiotics in the prior six weeks to CDI diagnosis. This finding is similar to recent regional studies conducted in Lebanon and Saudi Arabia to assess the risk factors for CDI revealed that the majority of participants diagnosed with CDI received antibiotics prior to the infection. ${ }^{20,21}$ A recent study in Egypt reported that patients with antibiotic treatment are at higher risk for CDI. ${ }^{22}$ The pathophysiology of antimicrobial agents disrupts the normal gastrointestinal flora, resulting in decreased body defenses against $C$. difficile, making it easier for $C$. difficile to produce toxins that cause CDI disease. ${ }^{23}$ Nearly all antibiotics have the potential to cause CDI with varying probabilities. Piperacillin-tazobactam, cephalosporins, vancomycin and aminoglycosides had the highest rates of positive CDI toxin tests. According to the National Institute of Health and Care Excellence (NICE), piperacillin-tazobactam is associated with a 1.5 times increase in CDI risk. ${ }^{24}$ Cephalosporins are among the highest risk categories of antibiotics that cause CDI. Fluoroquinolones, clindamycin, and penicillin are categorized as high-risk antibiotics for CDI. ${ }^{6}$ Although vancomycin and metronidazole are some of the treatment options for CDI, they have been associated with CDI, as shown in the results. There have been cases in which patients develop CDI after vancomycin or metronidazole. $^{25,26}$ Although aminoglycosides do not usually cause CDI, all antibiotics are associated with a risk of CDI. Other factors boost this risk, such as the duration of exposure, dose, number of antibiotics, and other patient factors. $^{23}$

PPIs and $\mathrm{H} 2$ receptor antagonists are used for many gastrointestinal disorders such as gastroesophageal reflux, heartburn, and peptic ulcer disease. Acid suppressors have been reported in many studies to increase the risk of CDI. A systematic review in Canada showed that acidsuppressing drugs are associated with an increased risk of CDI. ${ }^{27}$ In addition, many hypotheses have suggested that rising $\mathrm{PH}$ disrupts the normal flora; meanwhile, C. difficile spores are acid-resistant and survive. ${ }^{28}$ Other proposed hypotheses related to PPI include leukopenia ${ }^{29}$ and some antimicrobial properties of PPIs. ${ }^{29}$

In this study, approximately two-thirds of the patients used PPI, and half used a PPI for more than four days. The 
study results showed a clear association between PPI use and CDI, with a higher prevalence among patients on a PPI for more than four days. These results agree with an Egyptian study where patients who received PPIs were at higher risk of developing CDI. ${ }^{22} \mathrm{~A}$ meta-analysis also supports this observation where a PPI was associated with a $70 \%$ increase in CDI risk. ${ }^{30} \mathrm{H} 2$ blockers were only used by $4.9 \%$ of study participants and in comparison between $\mathrm{H} 2$ blockers and PPIs, the studies showed that $\mathrm{H} 2$ blockers have a lower risk of causing CDI than PPIs. ${ }^{31}$ PPI has been reported to increase the risk and severity of $\mathrm{CDI} .^{32}$ This study revealed that $84.6 \%$ of patients who had a severe form of CDI and $57.1 \%$ who had fulminant CDI were taking PPIs. Furthermore, PPI has been shown to increase the risk of hospital-acquired CDI (HA-CDI). ${ }^{33}$ These facts are very evident in our study; $93 \%$ of HACDI patients were taking PPIs.

CA and HA CDI prevalence were almost the same in this study, with $53.1 \%$ HA-CDI and $46.9 \%$ CA-CDI. This represents a change in the dynamics of CDI, which is known to be nosocomial. This increase in the prevalence of CA-CDI indicates a new disease pattern. This finding is similar to a USA study conducted in 2018. ${ }^{16}$ This change could be due to inappropriate selftreatment with antimicrobial agents without the supervision of physicians and the availability of PPIs and antacids secretory agents over the counter. However, HA-CDI patients suffer from a more severe form of the disease ${ }^{34}$ moreover, it is associated with higher mortality rates. ${ }^{35} \mathrm{CDI}$ is also classified by the IDSA guidelines based on the severity into mild, moderate, severe and fulminant. ${ }^{4}$ The current study showed that mild and moderate cases had the highest prevalence. Diarrhea was the chief complaint of most patients, with a prevalence of $90.1 \%$ in study participants, while $46.1 \%$ complained of abdominal pain.

Toxin A (TcdA) and toxin B (TcdB) are the primary markers for diagnosing CDI. Toxins are detected in the unformed stool of patients that grow in an anaerobic environment by tests such as glutamate dehydrogenase detection, toxin enzyme immunoassay, Nucleic Acid Amplification Tests (NAAT), and toxigenic stool culture. ${ }^{36}$ "The researchers recommended that NAAT be used as the primary diagnostic method for CDI." However, the optimal test for laboratory diagnosis remains controversial. ${ }^{37}$ These toxins cause symptoms of infection, ${ }^{38}$ as the results show in our study. Of the 593 participants, $17.7 \%$ had a positive $C$. difficile antigen, $13.3 \%$ had a positive toxin $\mathrm{A}$ test, and $13 \%$ had a positive result for toxin B. Based on the guidelines, treatment should be administered to patients with positive toxins $\mathrm{A}$ and $\mathrm{B}^{4}$ One important note is that toxin tests were performed on asymptomatic patients, which is unnecessary and not cost-effective.

According to the IDSA, vancomycin and fidaxomicin are the first treatment options for CDI, and metronidazole is used as an alternative if first-line options cannot be used. In the case of a fulminant form of CDI, vancomycin is still the first choice, but the route of administration differs and if ileus is present, IV metronidazole should be used. Of the patients with a confirmed diagnosis of CDI, $84.6 \%$ were treated as inpatients in-hospital settings. IDSA recommendation was only followed in $62 \%$ of patients due to formulary restrictions or an attractive medication cost of metronidazole. However, $94 \%$ of the patients responded to the treatment, regardless of the guideline recommendations.

\section{Limitations}

This retrospective descriptive study has potential limitations. First, we used previously documented patient files over three years. More than one doctor wrote notes about the same patient, so we read all the physician notes to answer questions and obtain accurate information. Thus, it was not easy to obtain data due to the hospital documentation system. Second, the sample size in our study was small due to different reasons, CDI has a low overall prevalence, and we also used information from one hospital. Because of the small sample size, we could not generalize the results to a broader population.

\section{Conclusion}

The prevalence, risk factors, and characteristics of CDI infections have similarities and differences between different countries. Although CDI prevalence is very low in Palestine, antibiotics, PPIs, and patient immune status continues to be the leading risk factors for acquiring CDI in hospitals or communities. C. difficile is one of the most important nosocomial infections worldwide. Nevertheless, the prevalence of CDI in community settings has been increasing. Most participants had a mildto-moderate form of the disease. In addition, there was an insignificant association between CDI and different genders or age groups. This finding cannot be generalized; however, more studies should be conducted on these factors to ensure association. Finally, complete 
patient assessments, awareness of CDI risk factors, appropriate utilization of PPIs, and antimicrobial stewardship are essential steps that healthcare providers can utilize to prevent or minimize CDI.

\section{Disclosure}

The authors report no conflicts of interest in this work.

\section{References}

1. Czepiel J, Dróżdż M, Pituch H, et al. Clostridium difficile infection: review. Eur J Clin Microbiol Infect Dis. 2019;38(7):1211-1221. doi:10.1007/s10096-019-03539-6

2. Balsells E, Shi T, Leese C, et al. Global burden of Clostridium difficile infections: a systematic review and meta-analysis. $J$ Glob Health. 2019;9:1.

3. Marley C, El Hahi Y, Ferreira G, Woods L, Ramirez Villaescusa A. Evaluation of a risk score to predict future Clostridium difficile disease using UK primary care and hospital data in clinical practice research datalink. Hum Vaccin Immunother. 2019;15(10):2475-2481. doi:10.1080/21645515.2019.1589288

4. McDonald LC, Gerding DN, Johnson S, et al. Clinical practice guidelines for Clostridium difficile infection in adults and children: 2017 update by the Infectious Diseases Society of America (IDSA) and Society for Healthcare Epidemiology of America (SHEA). Clin Infect Dis. 2018;66(7):e1-e48.

5. Thabit AK, Varugehese CA, Levine AR. Antibiotic use and duration in association with Clostridioides difficile infection in a tertiary academic medical center: a retrospective case-control study. Anaerobe. 2019;59:126-130. doi:10.1016/j.anaerobe.2019.06.016

6. Webb BJ, Subramanian A, Lopansri B, et al. Antibiotic exposure and risk for hospital-associated Clostridioides difficile infection. Antimicrob Agents Chemother. 2020;64(4):e02169-e02219. doi:10.1128/AAC.02169-19

7. Eze P, Balsells E, Kyaw MH, Nair H. Risk factors for Clostridium difficile infections - an overview of the evidence base and challenges in data synthesis. $J$ Glob Health. 2017;7(1):010417. doi:10.7189/ jogh.07.010417

8. Cui Y, Dong D, Zhang L, et al. Risk factors for Clostridioides difficile infection and colonization among patients admitted to an intensive care unit in Shanghai, China. BMC Infect Dis. 2019;19 (1):1. doi:10.1186/s12879-019-4603-1

9. Natarajan M, Rogers MA, Bundy J, et al. Gender differences in non-toxigenic Clostridium difficile colonization and risk of subsequent C. difficile infection. Clin Res Infect Dis. 2015;2:2.

10. Asempa T, Nicolau D. Clostridium difficile infection in the elderly: an update on management. Clin Interv Aging. 2017;12:1799-1809. doi:10.2147/CIA.S149089

11. Jump RL. Clostridium difficile infection in older adults. Aging Health. 2013;9(4):403-414. doi:10.2217/ahe.13.37

12. Miranda-Katz M, Parmar D, Dang R, Alabaster A, Greenhow TL. Epidemiology and risk factors for community associated Clostridioides difficile in children. $J$ Pediatr. 2020;221:99-106. doi:10.1016/j.jpeds.2020.02.005

13. Tschudin-Sutter S, Tamma PD, Naegeli AN, Speck KA, Milstone AM, Perl TM. Distinguishing community-associated from hospital-associated Clostridium difficile infections in children: implications for public health surveillance. Clin Infect Dis. 2013;57 (12):1665-1672. doi:10.1093/cid/cit581

14. Olsen MA, Stwalley D, Demont C, Dubberke ER. Increasing age has limited impact on risk of Clostridium difficile infection in an elderly population. Open Forum Infect Dis. 2018;5(7):7. doi:10.1093/ofid/ ofy 160
15. Kamboj M, Gennarelli R, Brite J, Sepkowitz K, Lipitz-Snyderman A. Risk for Clostridioides difficile infection among older adults with cancer. Emerg Infect Dis J. 2019;25(9):1683. doi:10.3201/ eid2509.181142

16. Revolinski SL, Munoz-Price LS. Clostridium difficile in immunocompromised hosts: a review of epidemiology, risk factors, treatment, and prevention. Clin Infect Dis. 2019;68(12):2144-2153. doi:10.1093/cid/ciy845

17. Solomon K. The host immune response to Clostridium difficile infection. Ther Adv Infect Dis. 2013;1(1):19-35. doi:10.1177/ 2049936112472173

18. Taking antibiotics increases your risk for C. diff infection. Center For Disease Control And Prevention; 2021. Available from: https://www. cdc.gov/cdiff/risk.html. Accessed June 15, 2021.

19. Teng C, Reveles KR, Obodozie-Ofoegbu OO, Frei CR. Clostridium difficile infection risk with important antibiotic classes: an analysis of the FDA adverse event reporting system. Int J Med Sci. 2019;16 (5):630-635. doi:10.7150/ijms.30739

20. Choucair J, Waked R, Haddad E, et al. Clostridioides difficile infections: epidemiology, correlations and treatment in a Lebanese cohort with use of ATLAS scoring. J Infect Dev Ctries. 2020;14 (12):1461-1465. doi:10.3855/jidc.13189

21. Alammari KM, Thabit AK. Characteristics of patients infected with Clostridioides difficile at a Saudi Tertiary Academic Medical Center and assessment of antibiotic duration. Gut Pathog. 2021;13(1):10. doi:10.1186/s13099-021-00405-9

22. Elgendy SG, Aly SA, Fathy R, Deaf EAE, Abu Faddan NH, Abdel Hameed MR. Clinical and microbial characterization of toxigenic Clostridium difficile isolated from antibiotic associated diarrhea in Egypt. Iran J Microbiol. 2020;12(4):296-304.

23. Castro I, Tasias M, Calabuig E, Salavert M. Doctor, my patient has CDI and should continue to receive antibiotics. The (unresolved) risk of recurrent CDI. Rev Esp Quimioter. 2019;32(Suppl 2):47-54.

24. NICE. Clostridium difficile infection: risk with broad-spectrum antibiotics; 2021. Available from: https://www.nice.org.uk/advice/ esmpb1/chapter/Key-points-from-the-evidence. Accessed November $1,2021$.

25. Bingley PJ, Harding GM. Clostridium difficile colitis following treatment with metronidazole and vancomycin. Postgrad Med J. 1987;63 (745):993-994. doi:10.1136/pgmj.63.745.993

26. Hecht JR, Olinger EJ. Clostridium difficile colitis secondary to intravenous vancomycin. Dig Dis Sci. 1989;34(1):148-149. doi:10.1007/ BF01536172

27. Leonard J, Marshall JK, Moayyedi P. Systematic review of the risk of enteric infection in patients taking acid suppression. $\mathrm{Am}$ $J$ Gastroenterol. 2007;102(9):2047-2056. doi:10.1111/j.15720241.2007.01275.x

28. Patil R, Blankenship L. Proton pump inhibitors and clostridium difficile infection: are we propagating an already rapidly growing healthcare problem? Gastroenterol Res. 2013;6(5):171. doi:10.4021/ gr575w

29. Gouraud A, Vochelle V, Descotes J, Vial T. Proton pump inhibitor-induced neutropenia. Clin Drug Investig. 2010;30 (8):559-563. doi:10.2165/11537230-000000000-00000

30. Leontiadis GI, Miller MA, Howden CW. How much do PPIs contribute to C. difficile infections? Am J Gastroenterol. 2012;107 (7):1020-1021. doi:10.1038/ajg.2012.174

31. Azab M, Doo L, Doo DH, et al. Comparison of the hospital-acquired Clostridium difficile Infection risk of using proton pump inhibitors versus histamine-2 receptor antagonists for prophylaxis and treatment of stress ulcers: a systematic review and meta-analysis. Gut Liver. 2017;11(6):781-788. doi:10.5009/gnl16568

32. Haider F, Raza N, Komar M, Rahman O, Sartorius J, Kirchner H. Proton pump inhibitor use elevates the risk of severe Clostridium difficile colitis. J Gastroenterol Hepatol Res. 2012;1(4):53-56. 
33. Lewis PO, Litchfield JM, Tharp JL, Garcia RM, Pourmorteza M, Reddy CM. Risk and severity of hospital-acquired Clostridium difficile infection in patients taking proton pump inhibitors. Pharmacotherapy. 2016;36(9):986-993. doi:10.1002/phar.1801

34. Juneau C, Mendias EP, Wagal N, et al. Community-acquired Clostridium difficile infection: awareness and clinical implications. J Nurse Pract. 2013;9(1):1-6. doi:10.1016/j.nurpra.2012.10.007

35. Oake N, Taljaard M, Van Walraven C, Wilson K, Roth V, Forster AJ. The effect of hospital-acquired clostridium difficile infection on in-hospital mortality. Arch Intern Med. 2010;170(20):1804-1810. doi:10.1001/archinternmed.2010.405
36. Zumla A. Mandell, Douglas, and Bennett's principles and practice of infectious diseases. Lancet Infect Dis. 2010;10(5):303-304. doi:10.1016/S1473-3099(10)70089-X

37. Wilkins TD, Lyerly DM. Clostridium difficile testing: after 20 years, still challenging. J Clin Microbiol. 2003;41(2):531-534. doi:10.1128/ JCM.41.2.531-534.2003

38. Voth DE, Ballard JD. Clostridium difficile toxins: mechanism of action and role in disease. Clin Microbiol Rev. 2005;18(2):247-263. doi:10.1128/CMR.18.2.247-263.2005

\section{Publish your work in this journal}

Infection and Drug Resistance is an international, peer-reviewed openaccess journal that focuses on the optimal treatment of infection (bacterial, fungal and viral) and the development and institution of preventive strategies to minimize the development and spread of resistance. The journal is specifically concerned with the epidemiology of antibiotic resistance and the mechanisms of resistance development and diffusion in both hospitals and the community. The manuscript management system is completely online and includes a very quick and fair peerreview system, which is all easy to use. Visit http://www.dovepress.com/ testimonials.php to read real quotes from published authors. 